\title{
Mycobacterium abscessus in patients with cystic fibrosis: low impact of inter-human transmission in Italy
}

To the Editor:

Mycobacterium abscessus is a rapidly growing nontuberculous mycobacterial species increasingly isolated worldwide [1]. It may cause chronic pulmonary infections, mainly in elderly patients with underlying bronchiectasis or chronic obstructive pulmonary disease, and it may also cause soft tissue, bone and joint infections [2]. M. abscessus has been isolated frequently from patients with cystic fibrosis (CF) [3], although its role in the decline of lung function remains unclear as it can be found both in patients with severe decrease in forced expiratory volume in 1 second (FEV1) and progressive worsening on computed tomography (CT) scan [4], and in asymptomatic patients. We investigated at the whole genome level M. abscessus isolated from all patients attending four Italian CF centres in the past decade with the aim of assessing the role of inter-human transmission.

The four centres are located in geographically distinct regions of Italy. All of the centres routinely perform sputum screening for mycobacteria using conventional protocols [5], together with extended incubation culture on Burkholderia cepacia selective agar (BCSA). The selective activity of BCSA for rapidly growing mycobacteria [6] allows the risk of missing M. abscessus through contamination or overgrowth of other bacterial species to be minimised. No special segregation policy was in force in any of the centres during the years covered by this study.

M. abscessus consists of three subspecies: M. abscessus subsp. abscessus, M. abscessus subsp. bolletii and M. abscessus subsp. massiliense [7]. We identified 306 isolates at the subspecies level by $r p o B$ gene sequencing [8]. In our study, $M$. abscessus subsp. abscessus accounted for $62 \%$ of isolates, in agreement with reported data [9]. Unexpectedly, M. abscessus subsp. bolletii was the second most prevalent subspecies (22\%), suggesting that this subspecies is not as rare as previously thought.

In total, 162 isolates were used for whole genome sequencing (WGS); one isolate was taken from each patient and at least two (the first and the last) if multiple isolates were found. In patients shown to be infected by more than one strain belonging either to the same or different subspecies, a representative proportion of isolates were selected for WGS, with the objective of differentiating persistent infections from occasional re-infections.

Cluster analysis of the isolates was performed by single nucleotide polymorphism (SNP) detection using an arbitrary cut-off based on the average difference of isolates collected in a short time interval from the same patient [10]. Reads were mapped to the M. abscessus ATCC19977 genome (NC_010397.1) using BWA (http://bio-bwa.sourceforge.net), and mappings refined with the GATK and Samtools toolkits. SNPs were called by Samtools and perl scripts with a minimum coverage of four reads in both forward and reverse orientation, with the four reads calling the allele with a phred score of at least 20 and allele frequency of $75 \%$. SNP positions were then combined, and positions with a reliable base call in at least $95 \%$ of the isolates were concatenated to a sequence alignment, excluding SNPs within a window of 12 bp from each other.

In 42 out of 48 patients with multiple isolates of $M$. abscessus over time, the average difference in SNPs was seven (range 0-22), a low number suggesting the persistence of the same strain and not a re-infection. In four patients, we isolated more than one subspecies of $M$. abscessus at different time points. In another

@ERSpublications

No outbreak of $M$. abscessus among Italian CF patients despite the presence of a widespread circulating clone http://ow.ly/oM9130cs6M0

Cite this article as: Tortoli E, Kohl TA, Trovato A, et al. Mycobacterium abscessus in patients with cystic fibrosis: low impact of inter-human transmission in Italy. Eur Respir J 2017; 50: 1602525 [https://doi.org/ $10.1183 / 13993003.02525-2016]$. 


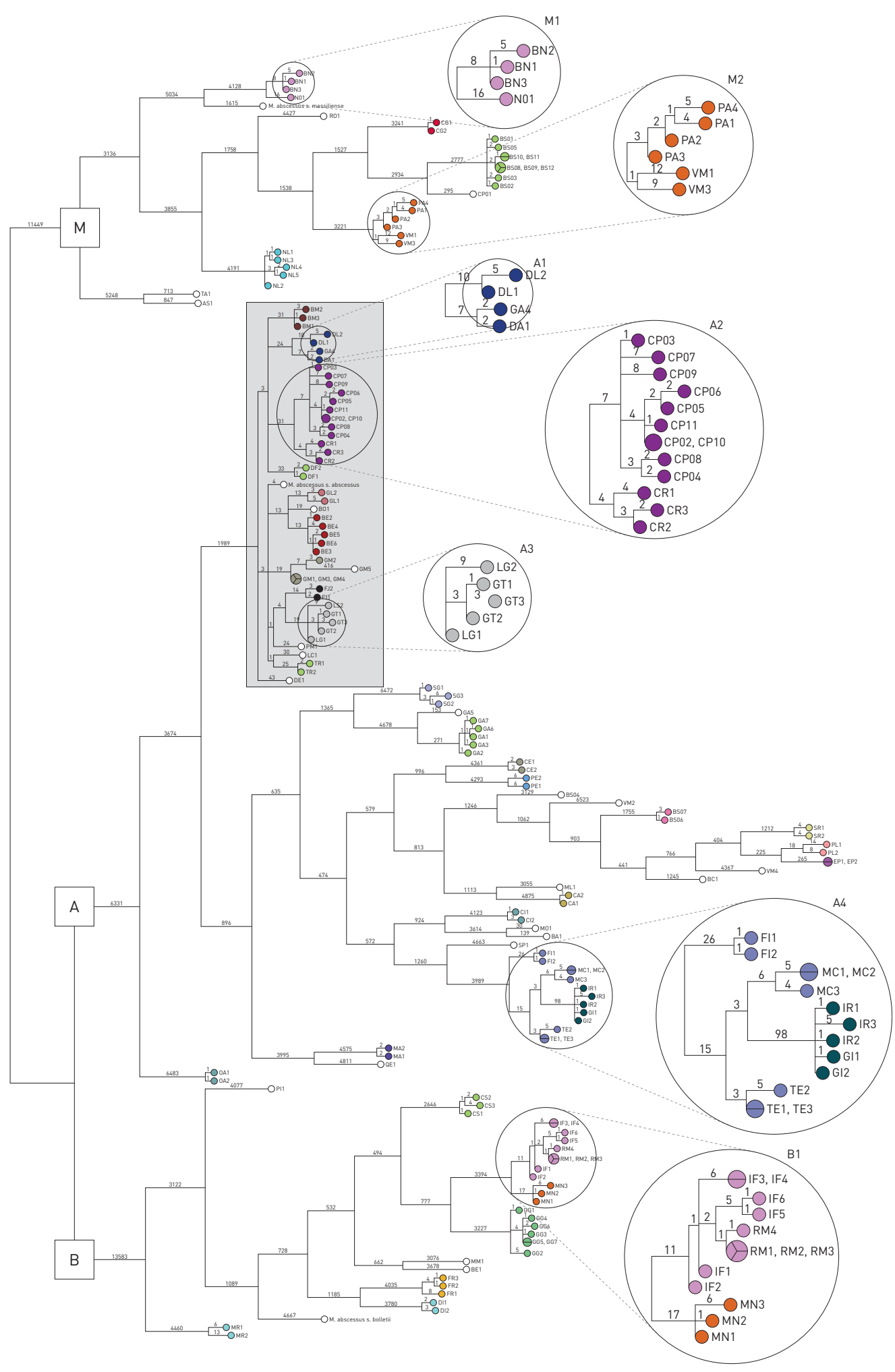

FIGURE 1 Maximum parsimony phylogenetic tree constructed on 110551 SNP positions of 162 clinical isolates and three reference strains of Mycobacterium abscessus on a logarithmic scale. Numbers on the branches indicate the number of distinct SNP positions between isolates. All isolates were grouped together las indicated by the same colourl with a maximum distance of 25 SNPs to the nearest group member. $M, A$ and $\mathrm{B}$ indicate the branches corresponding to the three subspecies: $M$. a. subsp. massiliense, $M$. $a$. subsp. abscessus and M. a. subsp. bolletii respectively. The grey shaded box highlights the dominating clone of $M$. abscessus subsp. abscessus. Clustered isolates are shown in circles M1, M2, A1, A2, A3, A4 and B1; each isolate is indicated by a two-letter identifier, unique to each patient, followed by a number representing the order of isolation. SNP: single nucleotide polymorphism. 
two patients, two unrelated strains of the same subspecies were detected. In almost all cases presenting more than one subspecies (5/6), we observed persistence of the same strain over time.

WGS confirmed the species identification performed by $r p o B$ Sanger sequencing in all cases except two strains that, despite the presence of a $r p o B$ sequence specific of $M$. abscessus subsp. abscessus, were assigned to $M$. abscessus subsp. massiliense by WGS.

Figure 1 shows the minimum parsimony tree of the 162 isolates analysed by WGS. Three main clusters corresponding to the subspecies are clearly identifiable (rectangles $\mathrm{A}, \mathrm{B}$ and $\mathrm{M}$ ). Within M. abscessus subsp. abscessus (figure 1A), we observed one group of clustered isolates (grey shading) originating from $15(25 \%)$ different patients, whereas the remaining isolates were dispersed. The median branch length of the clustered isolates was 21 SNPs (range 1-144).

A recent paper by BRYANT et al. analysing WGS of a vast number of $M$. abscessus isolated from patients with CF worldwide [11] reported the existence of at least three dominant clones responsible for at least three outbreaks postulated to be consequent to direct or fomite-mediated transmission. The analysis of our genomes, together with 30 of those reported by BRYANT et al. [11] (10 for each for the three major clusters: Abscessus 1 and 2 and Massiliense) revealed that Abscessus 1 overlapped the largest cluster identified in our collection (grey shading in figure 1). To avoid the risk of overlooking transmission episodes, we intentionally kept the threshold for flagging possible patient-to-patient transmission wide ( $\leqslant 30$ SNPs, with our same-patient difference being $\leqslant 21$ SNPs in $99 \%$ of the cases). By applying this threshold, we detected in the aforementioned cluster three possible patient-to-patient transmission episodes involving seven patients in total ( 3 in A1 and 2 each in A2 and A3). An additional cluster was identified within $M$. abscessus subsp. abscessus dispersed isolates, which involved five patients (A4). The number of SNPs confirmed possible occurrence of transmission for only two patients (IR and GI). Two clusters were detected within M. abscessus. subsp. massiliense (M1 and M2), each involving two patients, while one cluster (B1) involving three patients was present for M. abscessus subsp. bolletii.

We were able to collect information on attendance of each patient at the different centres included in this study. Of the seven "possible" transmission episodes identified by WGS analysis using a cut-off of SNPs difference $\leqslant 30$, only three (M1, M2 and B1 [patients IF and RM only]) involved patients who had attended the same CF centre at the same time. For the remaining four patients, transmission appears very unlikely based on the geographical location. In fact, these cases involved either patients attending centres located in different cities (A1), or couples of patients (one adult and one paediatric) referred to the same centre but cared for in different and distant locations of the clinic (A2, A3 and A4). One of our small clusters (A4), which included strains that were not very closely related, coincided with the Abscessus 2 of BRYANT et al. [11], while Massiliense was far from our M. abscessus subsp. massiliense genomes with the exception of one strain (CP01), isolated only once from one patient. Importantly, our investigation could exclude the occurrence in the past 12 years of major outbreaks of $M$. abscessus strains in any of the four Italian centres participating in this study. The discrepancy with the paper by BRYANT et al. [11] that reported large outbreaks in multiple countries may be explained by their much larger sample size; however, in an older study by BRYANT et al. [10], which was very similar to ours in terms of number of patients recruited, two large outbreaks of $M$. abscessus subsp. massiliense were reported in a single $\mathrm{CF}$ centre. Another explanation may be the very low incidence of $M$. abscessus subsp. massiliense in our study.

We conclude that the cluster Abscessus 1 described by BRYANT et al. [11] is present in Italy in the population of patients with CF. In addition, we identified very few highly clustered cases, each involving a maximum of three patients, for which the possibility of healthcare-related transmission could not be excluded. No major outbreaks were identified in our study in any of the participating centres. Our findings, far from minimising the importance of preventive infection control measures in CF centres, will hopefully reduce the concern in the CF community provoked by the emphasis given to the risk of inter-human transmission of $M$. abscessus.

Enrico Tortoli ${ }^{1}$, Thomas A. Kohl ${ }^{2}$, Alberto Trovato $^{1}$, Rossella Baldan ${ }^{1}$, Silvia Campana ${ }^{3}$, Lisa Cariani ${ }^{4}$, Carla Colombo ${ }^{5}$, Danila Costa $^{6}$, Simona Cristadoro, ${ }^{7}$ M. Clelia Di Serio ${ }^{8}$, Antonio Manca ${ }^{9}$, Giovanna Pizzamiglio $\oplus^{5}$, Paola M.V. Rancoita ${ }^{8}$, Gian Maria Rossolini ${ }^{10}$, Giovanni Taccetti ${ }^{3}$, Antonio Teri ${ }^{4}$, Stefan Niemann ${ }^{2}$ and Daniela M. Cirillo ${ }^{1}$

${ }^{1}$ Emerging Bacterial Pathogens Unit, IRCCS San Raffaele Scientific Institute, Milan, Italy. ${ }^{2}$ Molecular and Experimental Mycobacteriology, Forschungszentrum Borstel, Leibniz-Zentrum für Medizin und Biowissenschaften, Borstel, Germany. ${ }^{3}$ Regional Reference Center for Cystic Fibrosis, Meyer University Hospital, Florence, Italy. ${ }^{4}$ Cystic Fibrosis Microbiology Laboratory, IRCCS Ca' Granda, Milan, Italy. ${ }^{5}$ Cystic Fibrosis Center, IRCSS Ca' Granda, Milan, Italy. ${ }^{6}$ Microbiology Unit, Policlinico University Hospital, Bari, Italy. ${ }^{7}$ Cystic Fibrosis Center, Messina Hospital, Messina, Italy. ${ }^{8}$ Centre for Statistics in the Biomedical Sciences, Vita-Salute San Raffaele University, Milano, Italy. ${ }^{9}$ Reference Center for Cystic Fibrosis, Policlinico University Hospital, Bari, Italy. ${ }^{10}$ Microbiology and Virology Unit, Careggi University Hospital, Florence, Italy. 
Correspondence: E. Tortoli, Emerging Bacterial Pathogens Unit, IRCCS San Raffaele Scientific Institute, Milan, Italy. E-mail: e.tortoli.enrico@hsr.it

Received: Dec 222016 | Accepted after revision: April 072017

Support statement: This study was supported by the research grant FFC \#27/2014 from Fondazione Ricerca Fibrosi Cistica. Funding information for this article has been deposited with the Crossref Funder Registry.

Conflict of interest: None declared.

\section{References}

1 Mougari F, Guglielmetti L, Raskine L, et al. Infections caused by Mycobacterium abscessus: epidemiology, diagnostic tools and treatment. Expert Rev Anti Infect Ther 2016; 14: 1139-1154.

2 Tortoli E. Clinical manifestations of nontuberculous mycobacteria infections. Clin Microbiol Infect 2009; 15: 906-910.

3 Martiniano SL, Nick JA. Nontuberculous mycobacterial infections in cystic fibrosis. Clin Chest Med 2015; 36: 101-115.

4 Esther CR Jr, Esserman DA, Gilligan P, et al. Chronic Mycobacterium abscessus infection and lung function decline in cystic fibrosis. J Cyst Fibros 2010; 9: 117-123.

5 Garcia LS, Isenberg HD. Clinical Microbiology Procedures Handbook. Washington, ASM Press, 2010.

6 Esther CR Jr, Hoberman S, Fine J, et al. Detection of rapidly growing mycobacteria in routine cultures of samples from patients with cystic fibrosis. J Clin Microbiol 2011; 49: 1421-1425.

7 Tortoli E, Kohl TA, Brown-Elliott BA, et al. Emended description of Mycobacterium abscessus, Mycobacterium abscessus subs. abscessus, Mycobacterium abscessus subsp. bolletii and designation of Mycobacterium abscessus subsp. massiliense comb. nov. Int J Syst Evol Microbiol 2016; 66: 4471-4479.

8 Adékambi T, Colson P, Drancourt M. rpoB-based identification of nonpigmented and late-pigmenting rapidly growing mycobacteria. J Clin Microbiol 2003; 41: 5699-5708.

9 Macheras E, Konjek J, Roux AL, et al. Multilocus sequence typing scheme for the Mycobacterium abscessus complex. Res Microbiol 2014; 49: 491-499.

10 Bryant JM, Grogono DM, Greaves D, et al. Whole-genome sequencing to identify transmission of Mycobacterium abscessus between patients with cystic fibrosis: a retrospective cohort study. Lancet 2013; 381: 1551-1560.

11 Bryant JM, Grogono DM, Rodriguez-Rincon D, et al. Emergence and spread of a human-transmissible multidrug-resistant nontuberculous mycobacterium. Science 2016; 354: 751-757. 